\title{
VOLTERRA-STIELTJES INTEGRAL EQUATIONS WITH LINEAR CONSTRAINTS AND DISCONTINUOUS SOLUTIONS
}

BY CHAIM SAMUEL HÖNIG

Communicated by Alberto Calderón, November 11, 1974

$X$ and $Y$ denote Banach spaces; we consider systems of the form

$$
\begin{gathered}
y(t)-y\left(t_{0}\right)+\int_{t_{0}}^{t} d_{\sigma} K(t, \sigma) \cdot y(\sigma)=f(t)-f\left(t_{0}\right), \\
F[y]=c,
\end{gathered}
$$

where $y, f \in G([a, b], X)$ (the space of regulated functions $g:[a, b] \rightarrow X$, i.e., $g$ has only discontinuities of the first kind); $K \in G^{\text {uo }}$ (see §2) and $F \in$ $L[G([a, b], X), Y]$ (linear constraint). (K) includes linear Volterra integral equations, linear delay differential equations, differential equations $y^{\prime}+A^{\prime} y=f^{1}$, with the meaning that we have

$$
\text { (L) } y(t)-y(s)+\int_{s}^{t} d A(\sigma) \cdot y(\sigma)=f(t)-f(s) \text { for all } s, t \in[a, b] \text {. }
$$

In $\$ 2$ we give the existence of the resolvent for $(\mathrm{K})$ and in $\$ 3$ for $(\mathrm{L})$; in $\S 4$ we find the Green function for the system $(K),(F)$. The results of $\S 1$ are used in the proofs. All results of this announcement may be extended to open intervals and $Y$ a separated sequentially complete locally convex TVS.

The proofs will appear in [H.3].

1. A division of $[a, b]$ is a finite sequence $d: t_{0}=a<t_{1}<\cdots<t_{n}=b$. We write $|d|=n$ and $\Delta d=\sup _{1<i \leqslant n}\left|t_{i}-t_{i-1}\right|$. The set $D$ of all divisions of $[a, b]$ is ordered by refinement and $\lim _{d \in D} x_{d}$ denotes the limit according to the associated net. For $\alpha:[a, b] \rightarrow L(X, Y)$ and $f:[a, b] \rightarrow X$ we define the usual Riemann-Stieltjes operator integral

$$
\int_{a}^{b} d \alpha(t) \cdot f(t)=\lim _{\Delta d \rightarrow 0} \sum_{i=1}^{|d|}\left[\alpha\left(t_{i}\right)-\alpha\left(t_{i-1}\right)\right] \cdot f\left(\xi_{i}\right)
$$

where $\xi_{i} \in\left[t_{i-1}, t_{i}\right]$ (see [G], [H.1], [D]), and the interior integral

AMS (MOS) subject classifications (1970). Primary 34G05, 45 A05, 34A30, 45D05, 34B05; Secondary 26A45, 26A42, 28 A45. 


$$
\int_{a}^{b} d \alpha(t) \cdot f(t)=\lim _{d \in D} \sum_{i=1}^{|d|}\left[\alpha\left(t_{i}\right)-\alpha\left(t_{i-1}\right)\right] \cdot f\left(\xi_{i}\right)
$$

where $\left.\xi_{i}^{\cdot} \in\right] t_{i-1}, t_{i}[$ (see [K], [H, p. 96]), when these limits exist. The existence of the first integral implies the existence of the second one and reciprocally, if $\alpha$ and $f$ are bounded with no common discontinuity. We define

$$
S V[\alpha]=S V_{[a, b]}[\alpha]=\sup _{d \in D} S V_{d}[\alpha]
$$

where

$$
S V_{d}[\alpha]=\sup \left\{\left\|\sum_{i=1}^{|d|}\left[\alpha\left(t_{i}\right)-\alpha_{q}\left(t_{i-1}\right)\right] \cdot x_{i}\right\| \mid x_{i} \in X,\left\|x_{i}\right\| \leqslant 1\right\} .
$$

If $S V[\alpha]<\infty$ we say that $\alpha$ is of bounded semivariation and we write $\alpha \in$ $S V([a, b], L(X, Y))$; if we have further $\alpha(a)=0$ we write $\alpha \in S V_{0}([a, b], L(X, Y))$. For $u:[a, b] \rightarrow L(X, Y)$ we define $s[u]=\sup _{d \in D} s_{d}[u]$, where

$$
s_{d}[u]=\sup \left\{\left\|\sum_{i=0}^{|d|} u\left(t_{i}\right) \cdot x_{i}\right\| \mid x_{i} \in X,\left\|x_{i}\right\| \leqslant 1\right\}
$$

and we write $u \in s([a, b], L(X, Y))$ if $s[u]<\infty$. For $f \in G([a, b], X)$ we define $f_{-}(t)=f(t-)$ if $a \leqslant t \leqslant b$ and $f(a-)=0$; we write $f \in G_{-}([a, b], X)$ if $f_{-}=f$ and $f \in c_{0}([a, b], X)$ if $f_{-}=0$.

THEOREM 1. The mapping

$$
\begin{aligned}
(\alpha, u) & \in S V_{0}([a, b], L(X, Y)) \times s([a, b], L(X, Y)) \\
& \mapsto F=F_{\alpha}+F_{u} \in L[G([a, b], X), Y]
\end{aligned}
$$

defines a bicontinuous isomorphism of the first Banach space onto the second, where for $f \in G([a, b], X)$ we define

$$
F_{\alpha}[f]=\int_{a}^{b} d \alpha(t) \cdot f(t) \text { and } F_{u}[f]=\sum_{a \leqslant t \leqslant b} u(t) \cdot[f(t)-f(t-)] .
$$

We have $\left\|F_{\alpha}\right\|=S V[\alpha], \alpha(t) \cdot x=F\left[\chi_{1 a, t]} x\right]$ and $u(t) \cdot x=F\left[\chi_{\{t\}} x\right]$.

For $X=Y=R$ this theorem is due to Kaltenborn [K].

THEOREM 2. Given $\alpha \in S V([c, d], L(X, Y)), h:[c, d] \times[a, b] \rightarrow L(X)$ which is a regulated function in the first variable and uniformly of bounded semivariation in the second variable (i.e., $h^{t} \in S V([a, b], L(X))$ for every 
$t \in[c, d]$ and $\sup _{c<t<d} S V\left[h^{t}\right]<\infty$, where $\left.h^{t}(s)=h(t, s)\right)$ and $g \in G([a, b], X)$ we have $\bar{h} \in S V([a, b], L(X, Y))$, and $\tilde{g} \in G([c, d], X)$, where

$$
\bar{h}(s)=\int_{c}^{d} d \alpha(t) \cdot h(t, s) \text { and } \tilde{g}(t)=\int_{a}^{b} d_{s} h(t, s) \cdot g(s),
$$

and

$$
\int_{a}^{b} d_{s}\left[\int_{c}^{d} d \alpha(t) \circ h(t, s)\right] g(s)=\int_{c}^{d} d \alpha(t)\left[\int_{a}^{b} d s h(t, s) \cdot g(s)\right]
$$

If $[c, d]=[a, b]$ and $g$ is continuous we have the formula of Dirichlet

$$
\int_{a}^{b}\left[\int_{a}^{s} d \alpha(t) \circ h(t, s)\right] d g(s)=\int_{a}^{b} d \alpha(t) \cdot\left[\int_{t}^{b} h(t, s) d g(s)\right] .
$$

If $[c, d]=[a, b], \alpha \in A_{\bar{o}}$ (see §3) and $h \in G^{\text {uo }}$ (see §2) we have (2).

REMARK. (1) generalizes a theorem of Bray proved for $X=Y=R$ [B].

2. For $U:[a, b] \times[a, b] \rightarrow L(X)$ we consider the following properties $\left(\mathrm{SV}^{\mathbf{0}}\right)$

$$
\lim _{\delta \downarrow 0} S V_{[s-\delta, s+\delta]}\left[U^{t}\right]=0 \quad \text { for all } s, t \in[a, b],
$$

$$
\lim _{\delta \downarrow 0 u p, t} S V_{[s-\delta, s+\delta]}\left[U^{t}\right]=0 .
$$

We write $U \in G^{\text {uo }}$ if $U$ is bounded, regulated as a function of the first variable and satisfies $\left(S V^{\text {uo }}\right) \cdot G^{\text {uo }}$ is a Banach space when endowed with the norm $\|U\|=\|U\|+\sup _{a \leqslant t \leqslant b} S V\left[U^{t}\right]$.

THEOREM 3. Given $K \in G^{\mathrm{uo}}$ we have:

I. There is one and only one element $R \in G_{I}^{\mathrm{uo}}$ (i.e. $R \in G^{\mathrm{uo}}$ and $\left.R(t, t) \equiv I_{X}\right)$, the resolvent of $(\mathrm{K})$, such that

$$
R(t, s)=I_{X}-\int_{s}^{t} d_{\sigma} K(t, \sigma) \circ R(\sigma, s) \quad \text { for all } s, t \in[a, b]
$$

II. For every $f \in G([a, b], X)$ the equation $(\mathrm{K})$ with $y\left(t_{0}\right)=x$ has one and only one solution $y \in G([a, b], X)$ given by

$$
y(t)=R\left(t, t_{0}\right) x+\int_{t_{0}}^{t} R(t, \sigma) d f(\sigma)
$$

and $y$ depends continuously on $f, x$ and $K$.

III. If $K \in G_{0}^{\text {uo }}$ (i.e. $K \in G^{\text {uo }}$ and $K(t, t) \equiv 0$ ) we have

$$
R(t, s)=I_{X}+\int_{s}^{t} R(t, \sigma) \circ d_{\sigma} K(\sigma, s) \text { for all } s, t \in[a, b] \text {. }
$$


IV. The mapping $K \in G_{0}^{\text {uo }} \mapsto R \in G_{I}^{\text {uo }}$ is a bicontinuous (nonlinear) bijection from the first space onto the second.

REMARK. Theorem 3 remains true if we replace $G^{\text {uo }}$ by its subspace $E^{\text {uo }}$ of continuous functions, by its subspace $E^{\text {co }}$ of functions $U$ that satisfy $\left(\mathrm{SV}^{\mathrm{c}}\right) \quad \lim _{t \rightarrow t_{1}} S V\left[U^{t}-U^{t_{1}}\right]=0 \quad$ for every $t_{1} \in[a, b]$, by the corresponding spaces of functions of bounded variation, etc.

3. We now particularize Theorem 3 to (L). We fix a point $\bar{o} \in[a, b]$; given $A:[a, b] \rightarrow L(X)$ we write $A \in A_{\bar{o}}$ if $A(\bar{o})=0$ and if $A$ satisfies $\left(\mathrm{SV}^{\mathrm{o}}\right)$. $\left(\mathrm{SV}_{\mathrm{uo}}\right),\left(\mathrm{SV}_{\mathrm{o}}\right),\left(\mathrm{SV}_{\mathrm{c}}\right)$ denote the analogous for the first variable of the properties $\left(\mathrm{SV}^{\mathrm{uo}}\right),\left(\mathrm{SV}^{\mathrm{o}}\right),\left(\mathrm{SV}^{\mathrm{c}}\right)$ in the second variable. We say that $R:[a, b]$ $\times[a, b] \rightarrow L(X)$ is harmonic, and we write $R \in H$, if $R$ satisfies (SVuo), $\left(\mathrm{SV}^{\mathrm{c}}\right),\left(\mathrm{SV}_{\mathrm{uo}}\right),\left(\mathrm{SV}_{\mathrm{c}}\right)$ and

(o) $R(t, t) \equiv I_{X}, \quad R(t, \sigma) \circ R(\sigma, s)=R(t, s)$ for all $s, \sigma, t \in[a, b]$.

$H^{\text {co }}$ denotes $H$ with the topology induced by $E^{\text {uo }}$; analogously we define $H_{\text {co }}$. The next theorem extends Theorems 3.2 and 3.3 of [M].

THEOREM 4. A. Given $A \in \mathrm{A}_{\bar{o}}$ we have:

I. There is one and only one $R \in H$, the resolvent of $A$, such that

$$
R(t, s)=R(\tau, s)-\int_{\tau}^{t} d A(\tau) \circ R(\tau, s) \text { for all } s, \tau, t \in[a, b] .
$$

II. For every $f \in G([a, b], X)$ the equation (L) with $y(s)=x$ has one and only one solution $y \in G([a, b], X)$ given by

$$
y(t)=R(t, s) x+\int_{s}^{t} R(t, \sigma) d f(\sigma)
$$

and $y$ depends continuously on $f, x$ and $A$.

III. $A(t)=\int_{t}^{\bar{o}} d_{\sigma} R(\sigma, s) \circ R(s, \sigma)$ for all $s \in[a, b]$ and

$$
R(t, s)=R(t, \sigma)+\int_{\sigma}^{s} R(t, \tau) \circ d A(\tau) \text { for all } s, \sigma, t \in[a, b] .
$$

B. If $R:[a, b] \times[a, b] \rightarrow L(X)$ satisfies $(\mathrm{o})$ and $\left(S V_{\mathrm{o}}\right)$ then $R \in H$ and $R$ is the resolvent of $A$ given in III.

C. On $\mathrm{H}$ the topologies of $\mathrm{H}^{\mathrm{co}}$ and $\mathrm{H}_{\text {co }}$ coincide and the mapping $A \in A_{\bar{o}} \mapsto R \in H$ is a bicontinuous (nonlinear) bijection from the first space onto the second. 
4. We now consider the problem (K), (F) with $K \in E^{\text {uo }}$; we write $K[y]=f$ for $(K)$ and define $Y_{0}=F\left[K^{-1}(0)\right]$. Let $\alpha$ be associated to $F$ by Theorem 1; for $s \in[a, b]$ we define $J(s)=\int_{a}^{b} d \alpha(t) \circ R(t, s)$.

THEOREM 5. The following properties are equivalent:

(i) $y \equiv 0$ is the only solution of the system $K[y] \equiv 0, F[y]=0$.

(ii) $J\left(t_{0}\right): X \rightarrow Y_{0}$ is a continuous bijection.

From now on we suppose that the equivalent properties (i), (ii) are satisfied and that

$$
\left\{\int_{a}^{b} d \alpha(t) \cdot f(t) \mid f \in G([a, b], X)\right\}=Y_{0} .
$$

We define

$$
\bar{J}(t)=R\left(t, t_{0}\right) \circ J\left(t_{0}\right)^{-1}: Y_{0} \rightarrow X
$$

and

$$
\begin{aligned}
G(t, s)= & \bar{J}(t) \circ \int_{a}^{s} d \alpha(\tau) \circ R(\tau, s)-Y\left(s-t_{0}\right) \bar{J}(t) \circ J(s) \\
& +\left[Y\left(s-t_{0}\right)-Y(s-t)\right] R(t, s)
\end{aligned}
$$

Theorem 6. A.The system $K[y]=g, F[y]=c$ has a solution $y \in C([a, b], X)$ iff $(g, c) \in C([a, b], X) \times Y_{0}$; then this solution is

$$
y(t)=\bar{J}(t) c+\int_{a}^{b} G(t, s) d g(s)
$$

B. The system $K[y]=f, F[y]=c$ has a solution $y \in G([a, b], X)$ iff $c-F(f) \in Y_{0}$; then this solution is given by

$$
y(t)=f(t)+\bar{J}(t)[c-F(f)]-\int_{a}^{b} G(t, s) d_{s}\left[\int_{t_{0}}^{s} d_{\sigma} K(s, \sigma) \cdot f(\sigma)\right] .
$$

THEOREM 7. The Green function $G:[a, b] \times[a, b] \rightarrow L(X)$ has the following properties:

$\left(G_{0}\right) F\left[G_{s}\right]=0$ for every $s \in[a, b]$, where $G_{s}(t)=G(t, s)$.

$\left(G_{1}\right) G_{s}(t)-G_{s}\left(t_{0}\right)+\int_{t_{0}}^{t} d_{\sigma} K(t, \sigma) \circ G_{s}(\sigma)=\left[-Y(s-t)+Y\left(s-t_{0}\right)\right] I_{X}$.

$\left(\mathrm{G}_{2}\right) \widetilde{G}^{t}(s)+\int_{a}^{s} \widetilde{G}^{t}(\sigma) \circ d_{\sigma} K(\sigma, s)=\bar{J}(t) \circ \alpha(s)$ where

$$
\widetilde{G}(t, \sigma)=G(t, \sigma)+Y(\sigma-t) R(t, \sigma)+Y\left(\sigma-t_{0}\right)[\bar{J}(t) \circ J(\sigma)-R(t, \sigma)] \text {. }
$$

$\left(G_{3}\right)$ For every $s \in[a, b], G_{s}$ is continuous for $t \neq s$.

$\left(\mathrm{G}_{4}\right) G$ is uniformly of bounded semivariation in the second variable; $G(t, b) \equiv 0 ; G(t, a)=0$ for $a<t \leqslant b, G(a, a)=-I_{X}$. 


\section{REFERENCES}

B. H. E. Bray, Elementary properties of Stieltjes integral, Ann. of Math. 20 (1918/19), 177-186.

D. N. Dinculeanu, Vector measures, Internat. Series of Monographs in Pure and Appl. Math., vol. 95, Pergamon Press, Oxford; VEB Deutscher Verlag der Wissenschaften, Berlin, 1967. MR 34 \#6011b.

G. M. Gowurin, Über die Stielties Integration abstrakter Funktionen, Fund. Math. 27 (1936), 255-268.

H. T. H. Hildebrandt, Introduction to the theory of integration, Pure and Appl. Math., vol. 13, Academic Press, New York, 1963. MR 27 \#4900.

H.1. C. S. Honig, The Green function of a linear differential equation with a lateral condition, Bull. Amer. Math. Soc. 79 (1973), 587-593. MR 47 \#581.

H.2. - The abstract Riemann-Stieltjes integral and its applications to linear differential equations with generalized boundary conditions, Notas do Instituto de Matemática e Estatistica da Universidade de São Paulo, Série Matemática $n^{\circ}{ }_{1}, 1973$.

H.3. - , Volterra-Stieltjes integral equations, Math. Studies, vol. 16, NorthHolland, Amsterdam, 1975.

K. H. S. Kaltenborn, Linear functional operations on functions having discontinuities of the first kind, Bull. Amer. Math. Soc. 40 (1934), 702-708.

M. J. S. MacNerney, Stielties integrals in linear spaces, Ann. of Math. (2) 61 (1955), 354-367. MR 16, 716.

INSTITUTO DE MATEMÁTICA E ESTATISTICA, UNIVERSIDADE DE SÃO PAULO, SÃO PAULO, BRAZIL 